\title{
Descompresión orbitaria endoscópica transnasal en orbitopatía tiroidea severa
}

\author{
Transnasal endoscopic orbital decompression in severe thyroid orbitopathy
}

\author{
Miguel Henríquez A, Rodrigo Pacheco Z², Loreto Nicklas D¹, Guido Sepúlveda C³.
}

\begin{abstract}
RESUMEN
La orbitopatía tiroidea es una enfermedad autoinmune, en la que una reacción inflamatoria genera aumento de la presión orbitaria con protrusión de su contenido. A menudo es autolimitada y sus síntomas más frecuentes son retracción palpebral, exoftalmo y diplopía. Existen casos severos con compromiso de la agudeza visual por compresión del nervio óptico. El diagnóstico es clínico, pero debe complementarse con una tomografía computarizada. Su tratamiento depende de la gravedad y actividad de la enfermedad, siendo los procedimientos quirúrgicos, como la descompresión orbitaria, de elección en exoftalmo y neuropatía óptica compresiva. El pilar de tratamiento en la orbitopatía tiroidea severa es la cirugía descompresiva. Se han descrito múltiples técnicas, pero con limitaciones. La descompresión endoscópica transnasal, es considerada actualmente el procedimiento de elección, ya que permite una buena visualización de la pared medial, con resultados comparables y menores complicaciones, respecto a métodos tradicionales. Describimos un caso de oftalmopatía tiroidea severa, con exoftalmo, diplopía y disminución de la agudeza visual, en la que se realizó una descompresión endoscópica con muy buenos resultados.
\end{abstract}

Palabras clave: Orbitopatía tiroidea, descompresión orbitaria endoscópica, neuropatía óptica compresiva.

\begin{abstract}
Thyroid orbitopathy is an autoimmune disease in which an inflammatory reaction generates increased orbital pressure with protrusion of its contents. It is often selflimiting and its most frequent symptoms are eyelid retraction, exophthalmos and diplopia. There are severe cases with compromised visual acuity due to compression of the optic nerve. The diagnosis is clinical, but must be complemented with a computed tomography scan. Its treatment depends on the severity and activity of the disease and
\end{abstract}

\footnotetext{
1 Departamento de Especialidades Médicas, Universidad de La Frontera, Temuco. Servicio de Otorrinolaringología, Hospital Hernán Henríquez Aravena de Temuco, Chile.

2 Facultad de Medicina. Universidad de La Frontera, Temuco, Chile.

3 Servicio de Oftalmología, Hospital Hernán Henríquez Aravena de Temuco, Chile.

*Los autores declaran no tener conflictos de interés.

Recibido el 9 de junio, 2018. Aceptado el 2 de septiembre, 2018.
} 
the surgicals procedures such as orbital decompression is the best choice in exophthalmos and compressive optic neuropathy. The treatment in severe thyroid orbitopathy is decompressive surgery. Multiple techniques have been described, but with limitations. The transnasal endoscopic decompression is currently considered the gold standard, since it allows a good visualization of the medial wall with comparable results and less complications, compared to traditional methods. We present a case of severe thyroid ophthalmopathy, with exophthalmos, diplopia and decreased visual acuity, in which a transnasal endoscopic decompression was performed with very good outcomes.

Key words: Thyroid orbitopathy, endoscopic orbital decompression, compressive optic neuropathy.

\section{INTRODUCCIÓN}

La orbitopatía tiroidea es una enfermedad autoinmune que afecta la totalidad del globo ocular ${ }^{1,2}$. Complejos inmunes contra el receptor de tirotropina, presente en tiroides y órbita ${ }^{2}$, se unen a la musculatura extraocular, incitando una reacción inflamatoria que aumenta el volumen del contenido de la órbita, generando un aumento de la presión orbitaria con protrusión del globo ocular ${ }^{1,3,4}$. Está presente en $30 \%$ a $50 \%$ de los pacientes con enfermedad de Graves, siendo la manifestación extratiroidea más común $n^{2,3,5,6}$. Se estima una incidencia en la población general de 16 mujeres y 3 hombres por 100.000 personas al añ $0^{5,7}$, siendo más frecuente entre la quinta y sexta década de la vida ${ }^{2}$. Además se describe que en $20 \%$ a $60 \%$ existe historia familiar de enfermedad tiroidea ${ }^{8}$. Entre sus hallazgos más significativos encontramos inflamación de los contenidos orbitarios, depósitos de glicosaminoglicanos, infiltración linfocítica en músculos extraoculares y adipogénesis ${ }^{2-8}$. Participan factores genéticos, autoinmunes y ambientales. Los cambios que ocurren en la órbita se deben a una interacción de fibroblastos, activados por autoanticuerpos, y linfocitos T, la que se ve favorecida por factores ambientales, como el tabaco, que genera un riesgo 5 veces mayor a desarrollar orbitopatía tiroidea ${ }^{5,8}$. El $80 \%$ ocurre en pacientes hipertiroideos, $5 \%-10 \%$ en eutiroideos y $10 \%$ en hipotiroidismo autoinmune primario ${ }^{1}$. A menudo es autolimitada, pero una oftalmopatía tiroidea severa puede tener consecuencias significativas, como queratitis por exposición y neuropatía óptica compresiva¹. Los síntomas más frecuentes son retracción del párpado, observada en $80 \%$ a $90 \%$, asociado proptosis 0 exoftalmo. También puede producir diplopía por la restricción de movimientos oculares, lagoftalmo, sensación de cuerpo extraño, fotofobia, epífora, edema, eritema ${ }^{3}$ y compresión del nervio óptico, que puede producir pérdida progresiva de la agudeza visual, disminución de la visión a color y defectos en el campo visual, que se ve solo en casos severos, con una incidencia de $8,6 \% \%^{1,2}$. Distinguimos 2 fases: activa 0 inflamatoria e inactiva 0 estable, donde no ha habido actividad por 6 a 12 meses y se observa fibrosis residual. Están definidas por la escala CAS (Clinical Activity Score) de Mourits y "the Amsterdam Orbitopathy Group"3.

Del estudio de imágenes, particularmente útil al planificar una cirugía descompresiva 0 indicar tratamiento con radioterapia, tenemos que la TC (tomografía computarizada) es lo más utilizado, ya que tiene una sensibilidad mayor que la RM (resonancia magnética) en identificar fibrosis de músculos extraoculares ${ }^{5}$. La decisión de iniciar tratamiento y, a su vez, cuál alternativa terapéutica se empleará, depende de la gravedad y actividad de la enfermedad ${ }^{2}$. El enfoque de manejo debe ser bajo la clasificación VISA (Vision, Inflammation, Strabismus, and Appearance) de Dolman y Rootman, donde la neuropatía óptica es la primera prioridad ${ }^{7,9}$. El tratamiento de la actividad inflamatoria consiste en uso de corticoides y radioterapia, dejando el tratamiento quirúrgico para los cambios estéticos y funcionales ${ }^{2,3,6}$ que en términos generales son: descompresión orbitaria, corrección de estrabismo y anormalidades del párpado ${ }^{3}$. Son a su vez la primera de elección en neuropatía óptica compresiva, 
permitiendo recuperar agudeza visual, disminuir el exoftalmo y evitar o limitar la exposición córnea producida por lagoftalmo². La mayoría de estos tratamientos se realizan en la fase inactiva de forma electiva, pero si existe neuropatía óptica compresiva o ulceración corneal se realizan de urgencia $^{3}$. La descompresión orbitaria consiste en cualquier procedimiento quirúrgico que aumente el espacio orbital, pudiendo realizarse por remoción ósea, de grasa orbital o una combinación de ambos $^{2}$. La descompresión ósea implica remover una 0 más de las cuatro paredes orbitales, con una incisión en el periostio o periórbita, permitiendo que el contenido congestionado se expanda en los espacios adyacentes, disminuyendo la presión intraorbitaria, llevando a la retroposición ocular². Existen cuatro superficies disponibles: pared medial y posteromedial, pared anterolateral y posterolateral, y piso y techo orbital ${ }^{10}$.

Se han descrito diferentes métodos para realizar la descompresión ósea. El primero fue Dollinger ${ }^{1,3,6}$, quien abordó la pared lateral por fosa subtemporal, con resultados mínimos ya que existe un espacio limitado, asociándose además a proptosis pulsátil. Luego Naffziger ${ }^{1}$, sugirió un abordaje del techo orbital por fosa craneal anterior (transfrontal), el cual requería una craneotomía, con alto riesgo de meningitis y fístula de líquido cefalorraquídeo. También tenemos el método de Sewall ${ }^{1}$, quien sugirió acceder a la pared medial a través de una etmoidectomía externa y a Hirsch ${ }^{1}$, quien abordó el piso orbital con la técnica de Caldwell-Luc, encontrando un buen sitio para descompresión, debido al gran espacio para acomodar los contenidos orbitarios, pero con el riesgo de dañar el nervio infraorbitario, que se encuentra cerca del piso, produciendo entumecimiento facial. Posteriormente Walsh y Ogura ${ }^{1,3,6}$, unificaron las técnicas de descompresión de piso y pared medial, a través de un abordaje transantral, que evitó incisiones externas y fue ampliamente aceptado por sus resultados, pero se asociaba a alta incidencia de complicaciones posoperatorias, como diplopía e hipoestesia infraorbitaria ${ }^{1,3,6}$. Fue Kennedy en 1990, quien sugirió la descompresión endoscópica transnasal, que ha sido considerada el procedimiento de elección, ya que se asocia a una baja morbilidad ${ }^{1,3}$. Sugirió una esfenoetmoidectomía endonasal endoscópica. Identificó la pared medial de la órbita, la base del cráneo, el ápex orbital y el canal óptico. Su procedimiento incluye abrir el receso frontal, realizar una antrotomía meatal media, removiendo pared medial y piso orbital, abriendo posteriormente la periórbita ${ }^{1}$. Actualmente la técnica quirúrgica de preferencia es remover la pared medial y el piso orbital medial al nervio infraorbitario $^{2,11}$, ya que es un buen sitio de descompresión, porque tenemos los senos maxilares y etmoidales y no hay estructuras importantes asociadas, que se realiza principalmente por abordaje endonasal².

\section{CASO CLÍNICO}

Presentamos el caso de una paciente mujer de 44 años, con antecedentes de resistencia a la insulina, dislipidemia y bocio micronodular eutiroideo con anticuerpos TRAB positivos. Evaluada por Servicio de Oftalmología del Hospital Hernán Henríquez Aravena en mayo de 2016, por ptosis palpebral progresiva, edema, exoftalmo y dolor en ambas órbitas de 4 meses de evolución. Se observó dolor orbitario espontáneo y a los movimientos oculares extrínsecos, exoftalmo de ambas órbitas, diplopía a la supraversión ocular con reflejo pupilar conservado. Presión intraocular normal bilateral y exoftalmometría $16 \mathrm{~mm}$ OD (ojo derecho) y $18 \mathrm{~mm}$ OI (ojo izquierdo) respectivamente. Se confirmó oftalmopatía secundaria a enfermedad de Graves en fase inflamatoria CAS: 3 y se indica manejo con esteroides orales, recibiendo 2 ciclos de 6 pulsos de metilprednisolona, con mala respuesta.

Posterior a los pulsos presenta mayor exoftalmo (20 mm OD y $19 \mathrm{~mm}$ OI) con AV (agudeza visual) 20/20 bilateral. Se decide presentar a comité para manejo quirúrgico. Se indica tiroidectomía total, que se realizó en julio de 2017. Biopsia compatible con cáncer papilar de tiroides del lóbulo izquierdo. Poscirugía persiste con dolor ocular, ojo rojo, agregándose diplopía y pérdida de la AV. Exoftalmometría: $25 \mathrm{~mm}$ OD y $26 \mathrm{~mm}$ OI. AV $20 / 25$ OD y 20/40 OI. Se realizó RM de órbitas en agosto 2017 donde se observa engrosamiento de la musculatura extrínseca bilateral y simétrica. Se evalúa TC de órbitas, que se realizó en noviembre 2017 (Figuras 1 y 2): engrosamiento muscular no muy llamativo, elongación del nervio óptico de ambos ojos, sin compresión a nivel del ápex en 


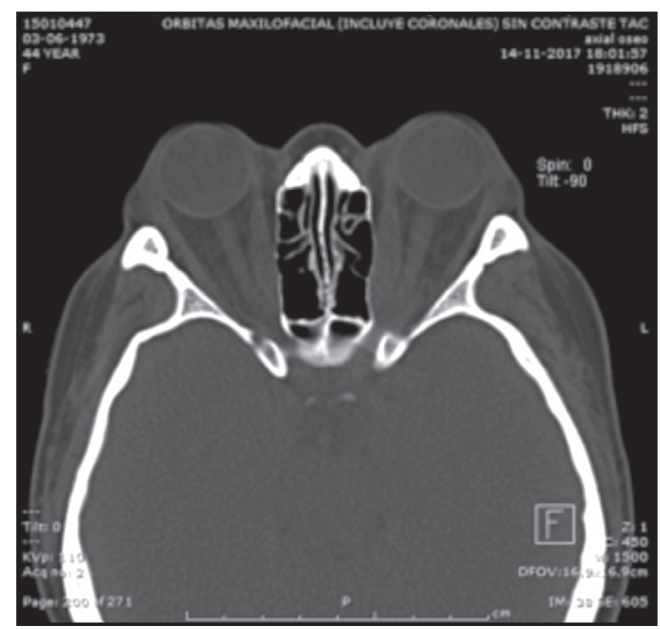

Figura 1. TC corte axial preoperatorio. Muestra exoftalmo con protrusión de contenido orbitario.

ambos ojos. Se solicitó evaluación por otorrinolaringología y se indicó descompresión endoscópica orbitaria por neuropatía óptica compresiva.

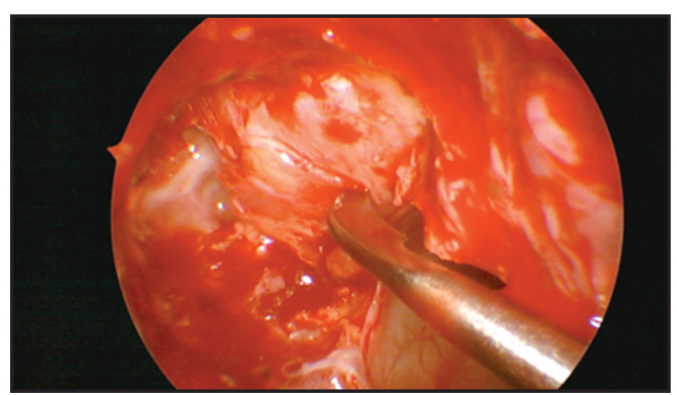

Figura 3. Imagen intraoperatoria de órbita izquierda, se realizan incisiones longitudinales sobre la periórbita.

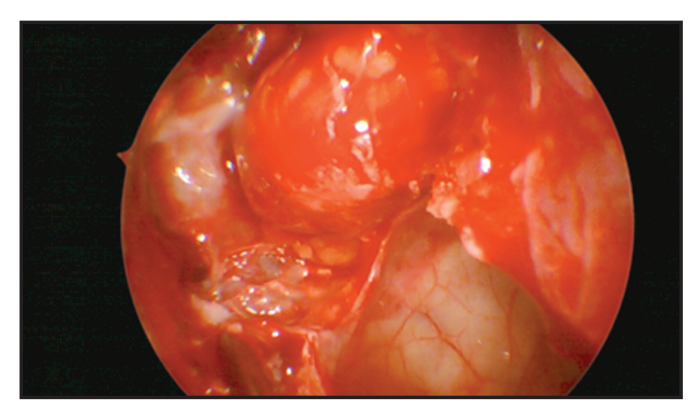

Figura 4. Órbita izquierda, con herniación orbitaria hacia fosa nasal tras resección de pared medial e inferior.

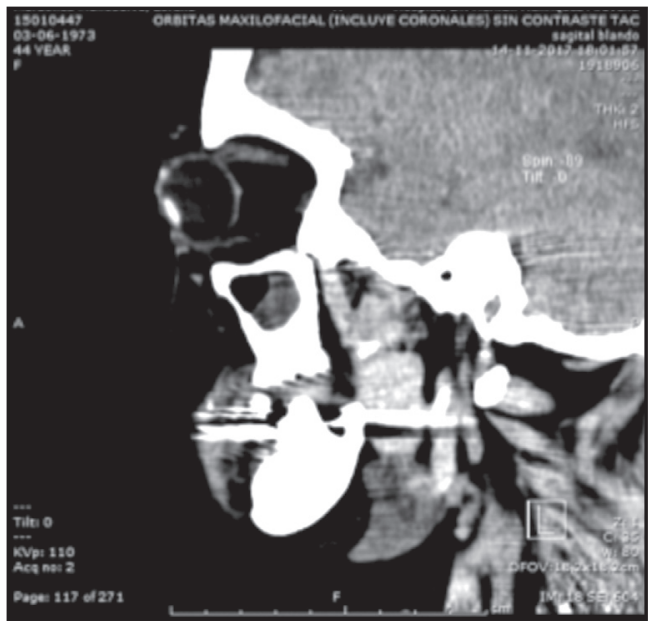

Figura 2. TC corte sagital de partes blandas preoperatorio. Muestra exoftalmo con protrusión de contenido orbitario.

En abril de 2018 se realizó una resección endoscópica bilateral, efectuando etmoidectomíaesfenoidectomía bilateral y una amplia antrostomía maxilar, con lo anterior se obtiene una mejor visualización de la órbita. Se resecó su pared medial e inferior, con incisiones en gran parte de la periórbita a través de cortes horizontales paralelos entre sí, extrayéndola y dejando sólo algunas franjas de seguridad, a fin de evitar diplopías (Figura 3). Observamos inmediatamente el prolapso de la grasa orbitaria hacia la cavidad nasal (Figuras 4 y 5), no fue necesario emplear tapones nasales. Tras finalizar la cirugía la paciente se trasladó a recuperación normal y posteriormente a una sala de complejidad estándar.

Evolucionó satisfactoriamente, sin diplopía, sin signos de fístula de líquido cerebroespinal

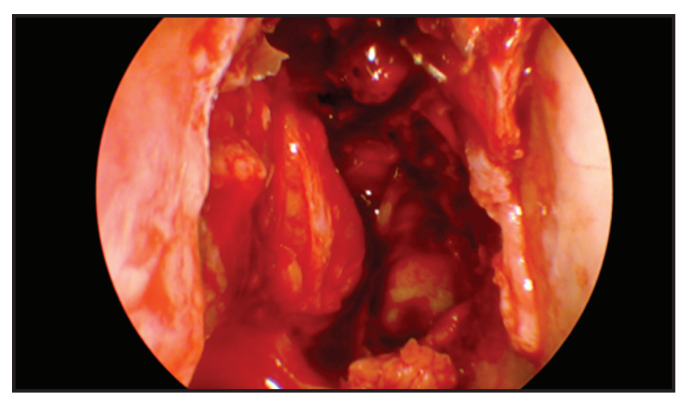

Figura 5. Órbita derecha, con herniación orbitaria hacia fosa nasal tras resección de pared medial e inferior. 
ni otras complicaciones por lo que fue dada de alta al día siguiente de la operación. Al alta se indican antibióticos (amoxicilina con ácido clavulánico) y una dosis decreciente de corticoides. Asistió a controles periódicos para realizar curaciones. Al mes posoperatorio fue controlada por servicio de oftalmología y otorrinolaringología. La paciente actualmente refiere diplopía leve al despertar y mejoría de la visión. Exoftalmometría $20 \mathrm{~mm}$ OD y $22 \mathrm{~mm}$ Ol. AV 20/20 OD y $20 / 25$ OI. Se tomó TC de órbitas de control en mayo 2018 (Figuras 6 y 7), en la que se observa la descompresión de la pared medial e inferior con protrusión de su contenido a la fosa endonasal y evidente disminución radiológica del exoftalmo.

\section{DISCUSIÓN}

El pilar de tratamiento para la orbitopatía tiroidea severa es la cirugía de descompresión, que se ha desarrollado por más de un siglo $0^{1,3,4}$. La técnica endoscópica con abordaje transnasal fue descrita inicialmente por Kennedy en 1990 en la búsqueda de nuevas alternativas quirúrgicas. Ha sido ampliamente usada, y se ha comparado con diversas técnicas como transantral y transconjuntival ${ }^{1}$. La primera tiene la desventaja del potencial daño en el nervio infraorbitario, edema facial posoperatorio y acceso limitado a la zona posterior de la pared medial. En el transconjuntival se limita la exposición y acceso a la zona posterior de regiones etmoidoesfenoidales. Actualmente, la endoscopía transnasal es el enfoque de elección, ya que tiene un buen acceso a la pared posteromedial de la órbita, permitiendo una descompresión máxima, una visualización de la pared medial que es superior a otras técnicas, con una descompresión óptima del nervio óptico, evitando una cicatriz externa y las morbilidades antes descritas ${ }^{1}$. Una desventaja del abordaje transnasal es que la descompresión del piso orbitario lateral al nervio infraorbitario y el piso orbitario anterior no son posibles debido a las limitaciones anatómicas, sin embargo, son contrapesadas por la excelente extensión medial y descompresión posterior proporcionada por este enfoque ${ }^{1}$.

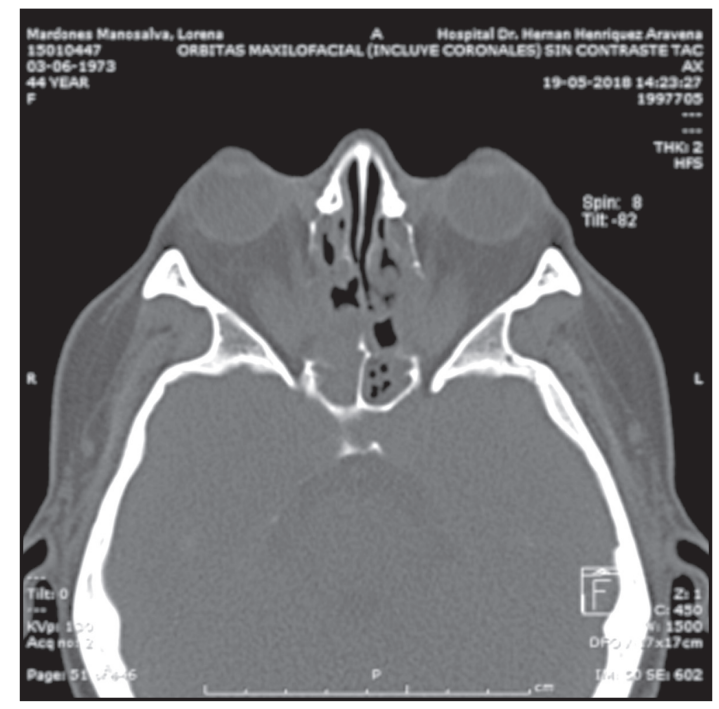

Figura 6. TC corte axial posoperatorio. Muestra contenidos orbitarios protruyendo a ambas fosas nasales.

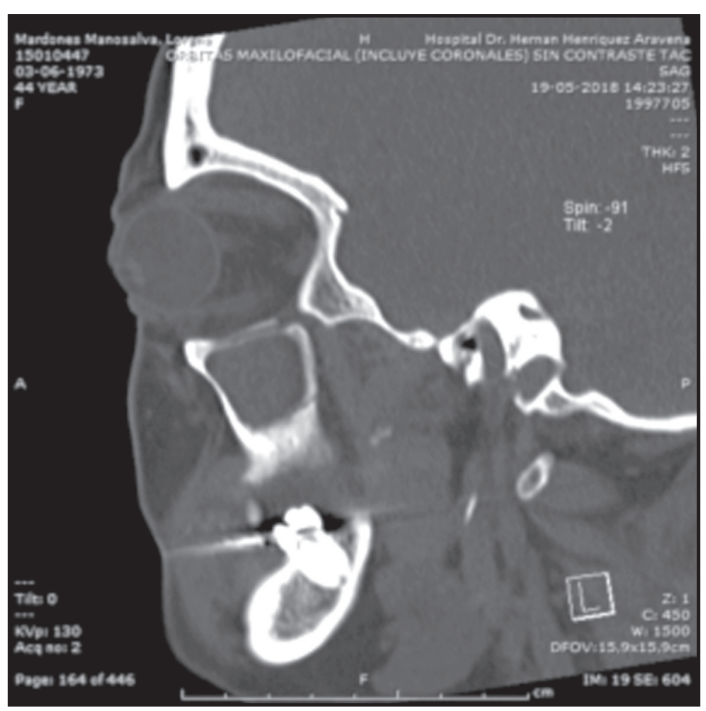

Figura 7. TC corte sagital posoperatorio. Muestra disminución de exoftalmo.

Se ha descrito que la técnica proporciona resultados comparables a los métodos tradicionales de la descompresión orbitaria evitando la morbilidad de la etmoidectomía externa 0 antrostomía de Caldwell-Luc, que abordan pared medial y piso orbitario respectivamente ${ }^{1}$. Warren y cols $^{12}$ en un estudio de 305 pacientes muestran una inci- 
dencia de $20 \%$ de hipoestesia posoperatoria del nervio infraorbitario sometidos a descompresión de Walsh-Ogura, de las cuales entre $4 \%$ a $5 \%$ fue permanente, además de $3,5 \%$ de fístula oroantral que requirió cirugía de cierre. También incluyen otras morbilidades como disestesia, edema facial y molestias dentales, las que son evitadas por el método de Kennedy. Pliego y cols ${ }^{13}$ compararon la técnica transantral de Walsh-Ogura y el método endoscópico transnasal de Kennedy, observando mayor incidencia de resultados adversos como diplopía y daño del nervio infraorbital en el primero.

Con respecto a las zonas que se deben resecar en nuestro caso resecamos ampliamente la pared medial e inferior, esta amplia antrostomía del seno maxilar además de permitir buena visualización evita que su drenaje se obstruya al protruir la grasa orbitaria. Así mismo se debe tener la precaución de dejar un margen superior de $1,5 \mathrm{~cm}$ para asegurar un adecuado drenaje frontoetmoidal. Kennedy describe en su técnica que al realizar el procedimiento hay que tener ciertas precauciones como que al remover el piso y pared medial en su zona posterior se debe dejar un pequeño borde de hueso anterior al canal óptico, que al actuar sobre el piso orbitario, éste debe ser medialmente al nervio infraorbitario y que se debe mantener la pared orbital medial en la región del receso frontal para evitar estenosis y obstrucción del seno frontal ${ }^{1,3,14}$.

Tal cual como describimos en nuestra técnica, al resecar el periostio se debe mantener franjas de este tejido para evitar unas de las complicaciones como es la diplopía'. Se ha informado que la diploplía inducida, es la complicación más común, planteando como factor causal la remoción de la pared posteromedial, exéresis del soporte inferomedial y el desplazamiento de la musculatura extraocular ${ }^{2}$. Es frecuente que luego de la cirugía no existan cambios respecto a este síntoma, además se puede desarrollar posoperatoriamente, e incluso puede empeorar ${ }^{1,6}$. Desanto y cols $^{15}$ observaron en descompresiones transantrales que la diplopía presente en $54 \%$ de los pacientes preoperatoriamente aumento a $79 \%$ en el posoperatorio. Otros estudios comparan las tres técnicas y observan desarrollo de esta alteración en 5,7\% luego de descompresión transconjuntival lateral y $40 \%$ luego de esta técnica vía transantral'1. Por lo anterior es que a los pacientes con diplopía se les recomienda someterse a des- compresión antes de cirugía muscular ${ }^{1}$. En nuestro caso solo se intervino piso y pared medial, pero el enfoque endoscópico transnasal puede combinarse con una orbitotomía lateral para proporcionar descompresión máxima, sin embargo, no se requiere el abordaje lateral cuando el exoftalmo no es grave, y el objetivo principal de la cirugía es la descompresión del nervio óptico en el ápice orbital, reservando combinar la resección de las 3 paredes en pacientes con alto grado de exoftalmo ${ }^{1,2}$. Una descompresión de la pared medial y piso se realizan en paciente con neuropatía óptica severa por acuñamiento apical de los músculos agrandados, mientras que la lateral produce reducción del exoftalmo y estrabismo.

Como mencionamos la máxima descompresión se obtiene con una combinación de la vía transnasal y orbitotomía lateral con un promedio de $5,7 \mathrm{~mm}$ de mejoría (rango de 4 a $7 \mathrm{~mm}$ ) en exoftalmometría, mientras que la descompresión nasal sola y Walsh-Ogura combinado con orbitotomía lateral tienen resultados semejantes con $4,5 \mathrm{~mm}(3-6 \mathrm{~mm})$ y $4,7 \mathrm{~mm}(3-6 \mathrm{~mm})$ respectivamente en exoftalmometría, sin haber correlación entre la proptosis preoperatoria y el grado de descompresión obtenido ${ }^{1}$. En experiencia de otros centros operados por oftalmopatía distiroidea con exoftalmo de $24 \mathrm{~mm}$ en promedio, se obtuvo una reducción de 4,6 $\mathrm{mm}$ en promedio (rango de 1-8 $\mathrm{mm}) 3,6$, lo que se compara con los resultados obtenidos por Kennedy y se asemeja a lo obtenido por nuestra paciente $5 \mathrm{~mm}$ en ojo derecho y $4 \mathrm{~mm}$ en ojo izquierdo al mes del posoperatorio.

En cuanto a la mejoría de la agudeza visual, se observó en pacientes con agudeza visual de 20/50 Snellen en promedio (rango de movimiento de manos a 20/25) una mejora, ya que el promedio posoperatorio fue 20/30 Snellen (rango 20/200 a 20/20). La AV mejoró en $70 \%$ de los pacientes, la que se relaciona con mayor reducción de propto$\mathrm{sis}^{16}$. Estos resultados son comparables con los de nuestra paciente que mejoró su AV en OD y OI de $20 / 25$ y 20/40 a 20/20 y 20/25 respectivamente. En cuanto a la descompresión orbitaria ósea 0 grasa, estudios muestran reducción satisfactoria del exoftalmo y una mejoría de visión, en caso de neuropatía óptica compresiva con ambas técnicas, sin diferencias significativas clínicas 0 en calidad de vida $^{2}$ pero se sabe que el combinar ambas técnicas se puede lograr una máxima descompresión. 


\section{CONCLUSIÓN}

La orbitopatía tiroidea es una enfermedad autoinmune que afecta la totalidad del globo ocular, generando un aumento de la presión orbitaria con protrusión del globo ocular, siendo la manifestación extratiroidea más común de la enfermedad de Graves. Uno de los pilares del tratamiento es la descompresión orbitaria, que es un procedimiento que se ha realizado por

\section{BIBLIOGRAFÍA}

1. Kennedy DW, Goodstein ML, Miller NR, Zinreich SJ. Endoscopic Transnasal Orbital Decompression. Archives of Otolaryngology-Head \& Neck Surgery 1990; 116: 275-82.

2. Boboridis KG, Bunce C. Surgical orbital decompression for thyroid eye disease. Cochrane Database of Systematic Reviews 2011; 12 : CD007630.

3. Tyler M, Zhang C, Saini A, Yao W. Cutting edge endonasal surgical approaches to thyroid ophthalmopathy. Laryngoscope Investigative Otolaryngology 2018; 3: 100-4.

4. Leung MK, Platt M, Metson R. Revision endoscopic orbital decompression in the management of Graves' orbitopathy. Otolaryngology-Head and Neck Surgery 2009; 141: 46-51.

5. Garrity J, Bahn R. Pathogenesis of Graves Ophthalmopathy: Implications for Prediction, Prevention, and Treatment. American Journal of Ophthalmology 2006; 142: 147-53.

6. Yao W, Sedaghat A, Yadav P, Fay A, Metson R. Orbital Decompression in the Endoscopic Age: The Modified Inferomedial Orbital Strut. Otolaryngology-Head and Neck Surgery 2016; 154: 963-9.

7. Bartalena L. Graves' Orbitopathy: Imperfect Treatments for a Rare Disease. European Thyroid Journal 2013; 2: 259-69.

8. Bahn RS, Heufelder AE. Pathogenesis of Graves' ophthalmopathy. NEJM 1993; 329: 1468-75. años, describiendo múltiples métodos. La técnica endoscópica nasal, considerada en la actualidad el procedimiento de elección, ofrece muy buenos resultados con menos efectos adversos descritos con las cirugías tradicionales. A través de la aplicación de nuestras técnicas y coordinándonos con otras especialidades, se ha ido ampliando el horizonte de nuestra especialidad, desarrollando procedimientos que cada vez obtienen mejores resultados clínicos.

9. Dolman P, Rootman J. VISA Classification for Graves Orbitopathy. Ophthalmic Plastic and Reconstructive Surgery 2006; 22: 319-24.

10. Goldberg RA. The Evolving Paradigm of Orbital Decompression Surgery. Archives of Ophthalmology 1998; 116: 95-6.

11. Mueller S, Miyake M, Lefebvre D, Freitag S, Bleier B. Long term impact of endoscopic orbital decompression on sinonasal specific quality of life. Laryngoscope 2018; 128: 785-8.

12. Warren JD, Spector JG, Burde R. Long-term follow up and recent observations on 305 cases of orbital decompression for dysthyroid orbitopathy. Laryngoscope 1989; 99: 35-40.

13. Pliego A, Miranda R, Vargas A, Marin Ja, Pena JF, Guerra J. Orbit decompression surgery in patients with exophthalmos caused by GravesBasedow disease. Gaceta Médica de México 2000; 136: 11-5.

14. Fuentealba D, Naser A, Oliva C, Ritacco L, Miranda G, Nazar R. Manejo endoscópico de osteoma etmoidal con extensión orbitaria: A propósito de un caso. Rev Otorrinolaringol Cir Cabeza Cuello 2017; 77: 281-8.

15. Desanto LW. The total rehabilitation of Graves' ophthalmopathy. Laryngoscope 1980; 90: 165278.

16. Leong S, Karkos P, Macewen C, White P. A systematic review of outcomes following surgical decompression for dysthyroid orbitopathy. Laryngoscope 2009; 119: 1106-15.

\footnotetext{
Correspondencia: Miguel Henríquez A.

Departamento de Especialidades Médicas. Universidad de la Frontera. Temuco

Claro Solar 115, Temuco, Chile

E mail: henmiguel@gmail.com
} 